\title{
Avaliação da sintomatologia ocular em pacientes com disfunção temporo-mandibular
}

\author{
Ocular symptom evaluation in patients with temporomandibular dysfunction
}

Gisele dos Santos Pereira ${ }^{(1)}$ Janaína Mesquita Duarte ${ }^{(1)}$ Eduardo Machado Vilela ${ }^{(2)}$

\section{RESUMO}

Objetivo: Avaliar a eficácia da placa neuromiorrelaxante em reduzir a sintomatologia oftalmológica de pacientes com disfunção temporo-mandibular (DTM) e constatar a possível correlação entre DTM e sintomatologia ocular.

Métodos: Foi realizada uma análise comparativa da evolução das sintomatologias da DTM e oculares em uma amostra de dezenove pacientes com DTM, sinais e sintomas oftalmológicos e ausência de patologia ocular. Esses pacientes foram submetidos a uma terapia reversível por meio do uso da placa neuromiorrelaxante.

Resultados: Observou-se que após o uso da placa durante um período maior (60 dias), o número de pacientes que apresentaram melhora de $100 \%$ ou que apresentaram sintomatologia discreta, tanto para DTM quanto para ocular, foi estatisticamente significativo. Já em intervalos de tempo menores (7-15, 15-30, 30-60 dias), o efeito da placa não foi significativo estatisticamente para reduzir a sintomatologia da DTM e ocular. A sintomatologia oftalmológica mais freqüente $(84,2 \%)$ encontrada nos pacientes com DTM da presente amostra foi dor ocular.

Conclusão: Há correlação entre DTM e sintomatologia ocular, visto que pacientes portadores desta disfunção que fizeram uso da terapia reversível, obtiveram melhora do quadro clínico tanto da DTM quanto da sintomatologia ocular num intervalo de tempo maior (7-60 dias).

Palavras-chave: DTM; Olho: sintomatologia; Placa neuromiorrelaxante.

\section{INTRODUÇÃ̃O}

Disfunção Temporo-Mandibular (DTM) se refere a um conjunto de desordens envolvendo os músculos mastigatórios, a articulação temporomandibular (ATM) ou ambos. Okeson ${ }^{10}$ e Steenks; Wijer ${ }^{13}$ afirmam que não existe um fator etiológico único que possa ser responsabilizado pela DTM, sendo, portanto, uma doença multifatorial. Várias doenças sistêmicas podem causar ou agravar DTM. Desordens do sistema imune como artrite reumatóide, artrite psoriática e lupus eritematoso sistêmico podem causar inflamação na ATM. Trauma extrínseco e deterioração artrítica são consideradas causas interrelacionadas ${ }^{3,5,8-13,18}$. De acordo com estatísticas publicadas no Journal of American Dental Association (JADA) em 1990, 44 a 99\% de problemas da ATM estão associados a trauma ${ }^{11}$. Sabe-se que DTM afeta mais mulheres do que homens no grupo etário de 20 a $30 \operatorname{anos}^{1,3,10,13}$. Segundo Okeson ${ }^{9}$ o aumento no nível do hormônio de Estudos da UFJF

Cirurgiã-Dentista graduada pela Universidade Federal de Juiz de Fora - MG

Professor da Faculdade de Odontologia da Universidade Federal de Juiz de Fora - MG

Os autores declaram que não possuem interesse financeiro no desenvolvimento ou publicação do referido estudo.

Endereço para correspondência: Dra. Gisela dos Santos Pereira. R. Mariano Procópio, 359. Centro Três Rios (RJ) CEP 25804-060. 
feminino estrógeno pode causar flacidez generalizada na ATM. Isso explica uma maior incidência de barulhos articulares em mulheres.

DTM é responsável pela maioria das dores orofaciais crônicas e são diagnosticadas através de seus sinais e sintomas característicos, tais como: ruído articular, cefaléia, artralgia, otalgia, mialgia, entre outros. A sintomatologia oftalmológica também pode estar presente, como: fotofobia, escurecimento da visão, espasmos dos músculos do olho, visão nublada, lacrimejamento, edema palpebral, hiperemia conjuntival, queimação e dor orbital 1-3,8-11,15, 16, 18. Agerberg; Inkapööl $1^{1}$ citam que os olhos estão entre os locais mais comuns de dor (20\%), assim como a face, garganta e pescoço. A localização anatômica das órbitas e globos oculares os predispõem a lesão em casos de traumas maxilofaciais ${ }^{17}$. O efeito chicote ("whiplash") com trauma direto na cabeça ou na mandíbula pode gerar, além de sintomas na ATM e no pescoço, cefaléia, mudanças visuais como sensibilidade à luz, visão escurecida, etc. Dor dentro e atrás do olho pode ser produzida por deslocamento da ATM, podendo causar fotofobia ${ }^{11}$.

"Trigger points", ou pontos álgicos no esternocleidomastóideo podem causar dor dentro, ao redor e abaixo dos olhos. Nos músculos cervicais posteriores, na área supra-orbital. No músculo occipitofrontal provocam dor reflexa atrás do olho. A palpação de pontos de gatilho no músculo masséter e temporal pode causar dor referida supra-orbital. Dor no músculo pterigóideo lateral pode se refletir para a região infra-orbital ${ }^{2,6,9,11}$.

Apesar da literatura se mostrar deficiente no que se refere a uma explicação da correlação entre DTM e a sintomatologia oftalmológica, a dor nos olhos e ao redor deles é relativamente comum ${ }^{1,2,9-11,16} \mathrm{e}$, a despeito de poder ser primária ou referida, raramente é o resultado de estímulos nocivos originados nos olhos, músculos extra-oculares ou no nervo óptico. Isto porque as funções sensitivas, nociceptivas e visuais das estruturas oculares não são paralelas. Existem terminações nervosas nociceptivas nos músculos extra-oculares, na bainha dural do nervo óptico e na periórbita que produzem a sensação de dor quando estiradas ${ }^{10}$.

Segundo Okeson ${ }^{9}$ a presença de dor profunda constante gera um efeito excitatório central. Quando este tipo de dor constante é sentida, o estímulo aferente convergido para o Sistema Nervoso Central (SNC) afeta outros neurônios.

Dependendo do interneurônio afetado (aferente, eferente ou autônomo) uma manifestação clínica distinta será apresentada: hiperalgia secundária (interneurônios aferentes), desenvolvimento de "trigger points" (interneurônios eferentes) e alteração da dilatação e a contração dos vasos sangüíneos quando há envolvimento de interneurônios autônomos. O resultado será um avermelhamento ou embranquecimento dos tecidos envolvidos. Os pacientes se queixam de pálpebras inchadas ou olhos secos ${ }^{9}$.

Uma estrutura anatômica ocular que parece estar relacionada à DTM são as veias oftálmicas ${ }^{4}$. De acordo com Weiner et al. ${ }^{16}$ há dados experimentais indicando alteração no fluxo sangüíneo em veias oftálmicas em pacientes com DTM.

Observando a relativa freqüência de pacientes com sintomatologia ocular, propusemo-nos fazer um estudo a respeito da possível correlação entre DTM e sintomatologia ocular. Para isso, realizamos uma revisão da literatura e estudo experimental dos pacientes que procuraram o Serviço de Diagnóstico e Orientação a Pacientes Portadores de DTM e que apresentaram sintomatologia ocular e DTM.

\section{METODOLOGIA}

Dentre os pacientes que procuraram o "Serviço ATM" da Faculdade de Odontologia da Universidade Federal de Juiz de Fora (FO - UFJF), no período de maio de 1997 a janeiro de 1998, foram selecionados 19 pacientes, sendo 14 mulheres e 5 homens, entre 12 e 58 anos. Estes apresentavam DTM, sintomatologia ocular e ausência de patologia ocular.

Uma carta endereçada aos oftalmologistas de Juiz de Fora foi entregue pessoalmente, informando-lhes sobre a pesquisa e solicitando o encaminhamento de pacientes que se encontrassem nas condições anteriormente citadas. Entretanto, não obtivemos o retorno esperado.

Para cada paciente foi feita uma avaliação odontológica, compreendendo queixa principal, história da doença atual, exame físico dental, muscular e articular. Foi ainda investigado sobre a freqüência e os fatores que acentuavam a sintomatologia prevalente, além de trauma prévio na região da ATM e/ou globo ocular.

Confirmado o diagnóstico de DTM, com sintomatologia ocular, os pacientes foram encaminhados ao oftalmologista com o intuito de fazer um exame oftalmológico, abrangendo acuidade visual, fundo de olho, pressão intra-ocular, meios transparentes, refração e mobilidade ocular.

Confirmado, dessa forma, o diagnóstico de DTM e ausência de patologia ocular, os pacientes que puderam se enquadrar na amostra foram submetidos a uma terapia reversível através do uso da placa neuromiorrelaxante. Foi realizada proservação com 7, 15, 30, 60 dias após instituído o tratamento, registrando a evolução das sintomatologias ocular e da DTM.

Para a análise clínica, foi estabelecido um sistema de escore, da seguinte forma:

A - Assintomático / S - Sintomatologia discreta / S1 Sintomatologia moderada / S2 - Sintomatologia acentuada.

Os pacientes da amostra tiveram acompanhamento psicológico durante o tratamento, ou seja, após uma consulta de diagnóstico psicológico, os pacientes receberam, durante o período proposto pela pesquisa (60 dias), acompanhamento do setor de Psicologia do "Serviço ATM" da FOUFJF através de três consultas de aconselhamento psicológico, numa abordagem visando orientação ao paciente quanto aos fatores emocionais (ex: estresse) que desencadeiam a DTM. 


\section{RESULTADOS}

A amostra do presente trabalho constou de 19 pacientes e seu perfil será apresentado nos gráficos que seguem (Gráficos $1,2$ e 3$)$.

$\mathrm{Na}$ tabela I verificou-se a prevalência de sintomatologia da DTM e sintomatologia ocular nos pacientes estudados. Observou-se que a cefaléia foi a sintomatologia da DTM mais prevalente $(94,7 \%)$ e que a dor ocular foi a que predominou em relação ao olho $(84,2 \%)$.

$\mathrm{Na}$ presente amostra, 36,8\% dos pacientes queixaram-se de sintomatologia de DTM e ocular no período noturno, enquanto $26,3 \%$ apresentaram sintomatologia pela manhã, $5,3 \%$ à tarde e $31,6 \%$ não souberam especificar a hora do dia em que a sintomatologia era mais freqüente.

Foram realizados testes de diferença de proporções para estudar a evolução das sintomatologias ocular e da DTM nos períodos de 7, 15, 30 e 60 dias, conforme Tabela II.

Na comparação dos tempos de 7 a 15 e 15 a 30 dias, não houve diferença significativa, ou seja, o uso da placa neuromiorrelaxante não alterou o estado do paciente. No período de 7 a 30 dias só houve diferença para a categoria A (melhora de $100 \%$ ), tanto para a sintomatologia de DTM quanto para a

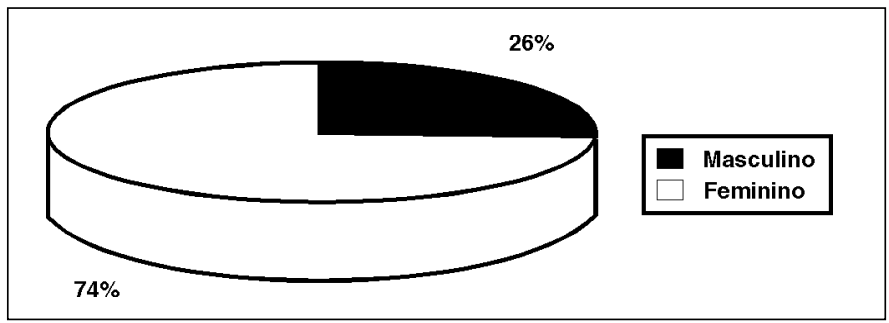

Gráfico 1 - Sexo dos pacientes

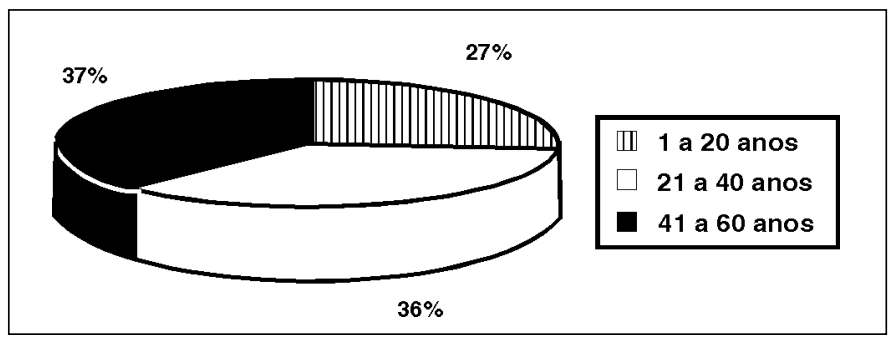

Gráfico 2 - Idade dos pacientes

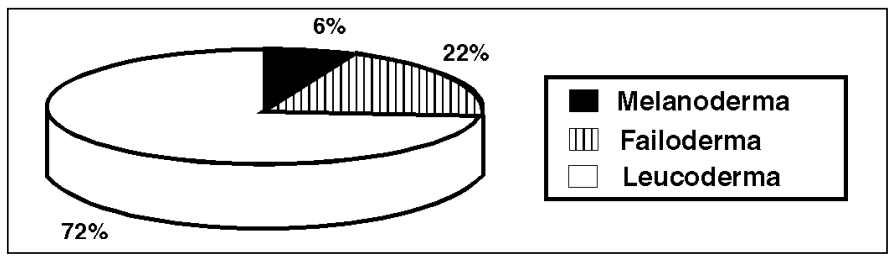

Gráfico 3 - Raça dos pacientes

\begin{tabular}{|c|c|c|c|}
\hline \multicolumn{2}{|c|}{ DTM } & \multicolumn{2}{|l|}{ OLHO } \\
\hline Cefaléia & $94,7 \%$ & Dor ocular & $84,2 \%$ \\
\hline Estalido & $68,4 \%$ & Lacrimejamento & $63,2 \%$ \\
\hline Tonteira & $57,9 \%$ & Hiperemia conjuntival & $52,6 \%$ \\
\hline Artralgia & $57,9 \%$ & Edema palpebral & $15,8 \%$ \\
\hline Dor facial & $47,4 \%$ & Outros** & $16,7 \%$ \\
\hline $\begin{array}{l}\text { Limitação da } \\
\text { abertura bucal }\end{array}$ & \multicolumn{3}{|l|}{$42,1 \%$} \\
\hline Outros* & \multicolumn{3}{|l|}{$78,9 \%$} \\
\hline \multicolumn{4}{|c|}{$\begin{array}{l}{ }^{*} \text { Neste ítem, incluem-se: dor cervical, otalgia, pressão no ouvido, zumbido, } \\
\text { bruxismo, edema na região temporo-mandibular e mialgia. }\end{array}$} \\
\hline \multicolumn{4}{|c|}{$\begin{array}{l}\text { ** Incluem-se: ardência, ressecamento ocular, fotofobia, pressão e sensação } \\
\text { de areia nos olhos. }\end{array}$} \\
\hline
\end{tabular}

ocular. Desta forma, foram utilizados os tempos de 7 dias e 60 dias, no que se refere às categorias estudadas. Houve diferença significativa para as categorias $\mathrm{S}$ e A tanto para a sintomatologia de DTM como para a sintomatologia ocular.

\section{DISCUSSÃO}

Apesar da literatura examinada mostrar-se deficiente no que se refere ao verdadeiro relacionamento existente entre a DTM e as manifestações oculares, esta relação é real. $\mathrm{Na}$ prática clínica, há muitos pacientes portadores de desordens da ATM que se queixam de sintomatologia ocular, apesar de serem normais do ponto de vista oftalmológico. Estes, após terem iniciado o tratamento para DTM, com a placa neuromiorrelaxante, a sintomatologia ocular diminuiu sensivelmente ou regrediu segundo relatos extraídos dos pacientes.

A sintomatologia ocular prevalente nos pacientes da amostra do trabalho realizado incluiu: dor ocular $(84,2 \%)$, lacrimejamento $(63,2 \%)$, hiperemia conjuntival $(52,6 \%)$, edema palpebral (15,8\%), e, perfazendo um total de 16,7\%, incluem-se: ardência, ressecamento ocular, fotofobia, pressão e sensação de areia nos olhos.

A descrição da dor ocular dada pelo paciente é usualmente completa, porque na maioria das vezes ela toma muito a atenção do paciente por horas, dias ou até anos; o que está de acordo com o presente trabalho, onde 52,6\% dos pacientes estudados relataram que a sintomatologia ocorria diariamente. A localização da dor pelo paciente, entretanto, é freqüentemente errônea e algum conhecimento de sinais e sintomas diferenciais permite ao examinador direcionar o exame de forma correta, bem como tomar decisões preliminares levando-se em consideração a urgência do caso ${ }^{7}$.

A hipótese etiológica da sintomatologia ocular em pacientes com DTM mais aceita na nossa opinião é o da dor profunda constante, gerando um efeito excitatório central.

Segundo Molina ${ }^{8}$, o fator mais importante para desencadear distúrbios na ATM é a falta de adaptação do paciente à uma 


\begin{tabular}{|c|c|c|c|c|c|c|c|c|}
\hline \multirow{3}{*}{ DIAS } & \multirow{2}{*}{\multicolumn{4}{|c|}{ DTM }} & \multirow{2}{*}{\multicolumn{4}{|c|}{ OCULAR }} \\
\hline & & & & & & & & \\
\hline & $\underline{\mathbf{S}}$ & S1 & S2 & A & $\underline{\mathbf{S}}$ & S1 & S2 & A \\
\hline \multirow[t]{2}{*}{$7 \times 60$} & $S$ & $\mathrm{~N}$ & $\mathrm{~N}$ & $S$ & $S$ & $\mathrm{~N}$ & $\mathrm{~N}$ & $S$ \\
\hline & 0,026 & 0,127 & 0,495 & 0,003 & 0,012 & 0,3156 & 0,656 & 0,023 \\
\hline \multirow[t]{2}{*}{$7 \times 30$} & $\mathrm{~N}$ & $S$ & $\mathrm{~N}$ & $S$ & $\mathrm{~N}$ & $\mathrm{~N}$ & $\mathrm{~N}$ & $S$ \\
\hline & 0,233 & 0,047 & 0,474 & 0,008 & 0,127 & 0,597 & 1,000 & 0,023 \\
\hline \multirow[t]{2}{*}{$7 \times 15$} & $\mathrm{~N}$ & $\mathrm{~N}$ & $\mathrm{~N}$ & $\mathrm{~N}$ & $\mathrm{~N}$ & $\mathrm{~N}$ & $\mathrm{~N}$ & $\mathrm{~N}$ \\
\hline & 0,445 & 0,474 & 1,000 & 0,445 & 0,474 & 1,000 & 1,000 & 0,507 \\
\hline \multirow[t]{2}{*}{$15 \times 60$} & $\mathrm{~N}$ & $\mathrm{~N}$ & $\mathrm{~N}$ & $N ?$ & $\mathrm{~N}$ & $\mathrm{~N}$ & $\mathrm{~N}$ & $\mathrm{~N}$ \\
\hline & 0,229 & 0,656 & 0,713 & 0,052 & 0,113 & 0,597 & 1,000 & 0,184 \\
\hline \multirow[t]{2}{*}{$15 \times 30$} & $\mathrm{~N}$ & $\mathrm{~N}$ & $\mathrm{~N}$ & $\mathrm{~N}$ & $\mathrm{~N}$ & $\mathrm{~N}$ & $\mathrm{~N}$ & $\mathrm{~N}$ \\
\hline & 1,000 & 0,337 & 0,713 & 0,104 & 0,656 & 1,000 & 0,656 & 0,184 \\
\hline \multirow[t]{2}{*}{$30 \times 60$} & $\mathrm{~N}$ & $\mathrm{~N}$ & $\mathrm{~N}$ & $\mathrm{~N}$ & $\mathrm{~N}$ & $\mathrm{~N}$ & $\mathrm{~N}$ & $\mathrm{~N}$ \\
\hline & 0,468 & 1,000 & 0,691 & 1,000 & 0,468 & 1,000 & 1,000 & 0,713 \\
\hline
\end{tabular}

oclusão que não é ideal para todos os mecanismos de adaptação. Os tipos de oclusão mais freqüentes nos indivíduos com DTM e músculos associados são aqueles com muita discrepância entre oclusão e relação cêntrica, com contatos e interferências no lado de equilíbrio, e oclusões onde se altera a dimensão vertical.

Pudemos confirmar este fato no presente estudo, onde $89,5 \%$ dos pacientes da amostra apresentaram oclusão cêntrica diferente de relação cêntrica. $68,4 \%$ dos pacientes estudados apresentaram interferência oclusal nos movimentos de lateralidade esquerda a direita.

Segundo Agerberg; Inkapööl ${ }^{1}$, os músculos mastigatórios mais freqüentemente sensíveis à palpação são o músculo pterigóideo lateral (34\%) e o músculo temporal $(27 \%)$. No atual estudo, apresentaram-se sensíveis à palpação e sem alteração de volume os músculos pterigóideo lateral em $57,9 \%$ dos pacientes, o esternocleidomastóideo em 42,1\%, o temporal em $36,8 \%$, o masséter em $26,3 \%$ e o pterigóideo medial em 5,3\% dos pacientes.

De acordo com Steenks; Wijer ${ }^{13}$, são considerados sinais e sintomas clássicos de DTM: ruídos na articulação, limitação dos movimentos e/ou desvios dos movimentos da mandíbula, dor a nível da ATM e/ou dos músculos mastigatórios. Molina ${ }^{8}$, Okeson ${ }^{9}$, Shankland ${ }^{11}$ e Turley ${ }^{14}$, citam que o sintoma mais freqüentemente referido nos casos de DTM é a presença de estalidos e/ou ruídos crepitantes em uma ou ambas ATM's durante os movimentos da mandíbula. $\mathrm{O}$ achado clínico mais freqüente no estudo de Agerberg; Inkapööl ${ }^{1}$ foi som da ATM $(58 \%)$. Cefaléias recorrentes também foram freqüentemente reportadas pelos pacientes $(43 \%)$.

No presente estudo, a sintomatologia inicial mais comumente reportada pelos pacientes foi cefaléia $(94,7 \%)$, seguida de estalido $(68,4 \%)$, artralgia e tonteira $(57,9 \%)$, dor facial $(47,4 \%)$ e limitação da abertura bucal $(42,1 \%)$.

O perfil da amostra deste estudo está de acordo com a literatura apresentada, onde as mulheres apresentam maior freqüência de sintomatologia de DTM que homens. A freqüência do sexo feminino foi de $73,7 \%$ e do sexo masculino, $26,3 \%$.

Num estudo comparativo da evolução das sintomatologias da DTM e ocular, pudemos observar que no intervalo de tempo maior 7-60 dias, obtivemos diferença significativa para um nível de significância de 5\% nas categorias A (assintomático) e S (sintomatologia discreta), tanto para DTM como para a sintomatologia ocular.

Comparando-se os intervalos de tempo menores, 7-15 e 15-30 dias, não houve diferença significativa, ou seja, o uso da placa neuromiorrelaxante não alterou o estado do paciente. No intervalo de 7-30 dias, houve diferença significativa para as categorias S1 (sintomatologia moderada) e A no que se refere a sintomatologia de DTM e, para a categoria A, em relação à sintomatologia ocular. Isto pode ser explicado pelo número reduzido da amostra, mediante a dificuldade de se encontrar pacientes que se enquadrassem no perfil da amostra proposto pelas autoras.

Sugere-se que novas pesquisas sejam feitas utilizando-se uma amostra maior a fim de que a correlação discutida possa ser mais uma vez confirmada e que a classe médica oftalmológica se torne ciente de tal correlação e possa, dessa forma, encaminhar o paciente para a correta terapia.

\section{SUMMARY}

Purpose: To evaluate if the neuromuscular relaxing plate is effective in reducing the ophthalmic symptoms of patients with temporomandibular dysfunction (TMD) and to observe the possible correlation between TMD and ocular symptoms.

Methods: A comparative analysis of TMD symptoms and ocular symptoms was performed in a sample of nineteen patients with TMD, ophthalmic signs and symptoms and absence of ocular diseases. Those patients were submitted to a reversible therapy using a neuromuscular relaxing plate.

Results: It was observed that after the use of the plate during 
a longer period of time (60 days) the number of patients who showed $100 \%$ improvement or mild symptoms was statistically significant for both TMD and ocular symptoms. In contrast, in short periods of time (7-15, 15-30, 30-60 days) the plate's effectivity was not statistically significant to reduce TMD and ocular symptoms. The most frequent ophthalmic symptom (84.2\%) shown by the patients with TMD of the sample was ocular pain.

Conclusion: There is a correlation between TMD and ocular symptoms, because the improvement of the clinical status of the patients with TMD who used the reversible therapy, was statistically significant for both TMD and ocular symptoms in a longer period of time (7-60 days).

Keywords: TMD; Eye: symptoms; Neuromuscular relaxing plate.

\section{AGRADECIMENTOS}

À Dra. Gislaine Sanglard da Silva, pelos exames oftalmológicos.

\section{REFERÊNCIAS BIBLIOGRÁAFICAS}

1. Agerberg G e Inkapöö I. Craniomandibular Disorders in Urban Swedish Population. Journal of Craniomandibular Disorders: Facial \& Oral Pain, Sweden 1990;4(3):154-64
2. Arlen H. Otolaryngologic Manifestations of Craniomandibular Disorders Dental Clinics of North America, New Jersey 1983;27(3):523-6.

3. Fricton JR. Recent Advances in Temporomandibular Disorders and Orofacial Pain. Journal of American Dental Association, USA 1991;122(11):25-32.

4. Gardner GO. Anatomia. Ed. Guanabara Koogan, $4^{\circ}$ edição, Philadelphia 1988.

5. Gelb $\mathrm{H}$ e Bernstein, J. Clinical Evaluation of Two Hundred Patients with Temporomandibular Joint Syndrome. The Journal of Prosthetic Dentistry, New York 1993;49(2):234-43.

6. Gray RJM et al. A Clinical Approach to Temporomandibular Disorders. Examination of the Articulatory System: The Muscles. British Dental Journal, Manchester 1994;177(1):25-8.

7. Kalina RE e Orcutt JC. Capítulo Parte de Monografia: Ocular and Periocular Pain. I.N.: The Management of Pain, Oxford; 759-68.

8. Molina OF. Fisiopatologia Craniomandibular (Oclusão e ATM). São Paulo $1989 ; 595 \mathrm{p}$.

9. Okeson JP. Fundamentos de Oclusão e DTM. Ed. Artes Médicas, $2^{\circ}$ edição, São Paulo 1992; 449 p.

10. Okeson JP. Dor Orofacial. Guia de Avaliação, Diagnóstico e Tratamento. Ed. Quintessence, 1 edição, São Paulo 1998.

11. Shankland WE. TMJ Headaches Dentistry Diseases and Face Alternative Medicine.

12. Sicher H et al. Anatomia Bucal. Ed. Guanabara Koogan, $6^{\circ}$ edição, São Paulo 1997; $511 \mathrm{p}$

13. Steenks MH e Wijer A. Disfunção da Articulação Temporomandibular do Ponto de Vista da Fisioterapia e da Odontologia. Diagnóstico e Tratamento. Livraria Santos Editora Ltda., 1 edição, São Paulo 1996.

14. Turley PK. Update on Temporomandibular Dysfunction, PCSO Bulletin Spring 1991;52-5

15. Weber JM. TMJ Syndrome: A Possible Cause of Unexplained Eye Problems, January $1984 ; 20 / 20$

16. Weiner LB et al. Monitory Ocular Changes that May Accompany Use Of Dental Appliances and/or Osteopathic Craniosacral Manipulations in the Treatment of TMJ and Related Problems. The Journal of Craniomandibular Practices, July 1987;5(3):278-85.

17. Wesseberg GA et al. Ophthalmologic Considerations in Maxillofacial Trauma Anatomy and Diagnostic Evaluations. Int J Oral Surg 1981;19:236-46.

18. Wijer A et al. Temporomandibular and Cervical Spine Disorders. Self-Reported Signs and Symptons. Lippincott-Raven Publishers 1996;21(14):1639-46.

\section{Simpósio da Sociedade Brasileira de Glaucoma}

\section{7 a 9 de Junho de 2001}

\section{Minascentro - Belo Horizonte - MG}

INFORMAÇÕES: Consult Comunicação e Marketin

Av. Agusto de Lima, 479 - Ci. 1611

CEP 30190-000 - Belo Horizonte - MG

Telefax: (00xx31) 274-1550

E-mail: comunica@consultcom.com.br

http://www.cbo.com.br/abo 